\title{
Missions du futur et nouveaux concepts en propulsion plasma
}

Laurent Garrigues (laurent.garrigues@laplace.univ-tlse.fr), Freddy Gaboriau, Alexandre Guglielmi et Jean-Pierre Boeuf Laboratoire plasma et conversion d'énergie (LAPLACE, UMR CNRS 5213)

Université Toulouse-Paul Sabatier, 118 route de Narbonne, 31062 Toulouse Cedex 9

L'intérêt d'utiliser des particules chargées accélérées à de grandes vitesses pour propulser un engin spatial a germé dans la tête de C. Tsiolkovski (1857-1935), qui a posé les fondements de l'astronautique dans son ouvrage de 1903. Il a fallu néanmoins attendre la course à l'espace durant la guerre froide pour voir émerger les premiers concepts de propulseurs à plasma, puis leur utilisation sur les satellites et les premières sondes d'exploration à la fin du $X X^{\mathrm{e}}$ siècle.

Le $\mathrm{XXI}^{\mathrm{e}}$ siècle ouvre la voie à de nouveaux concepts pour des plateformes de satellites toujours plus massives, des sondes d'exploration envoyées toujours plus loin, ou des vols habités pour l'exploration de la planète Mars.

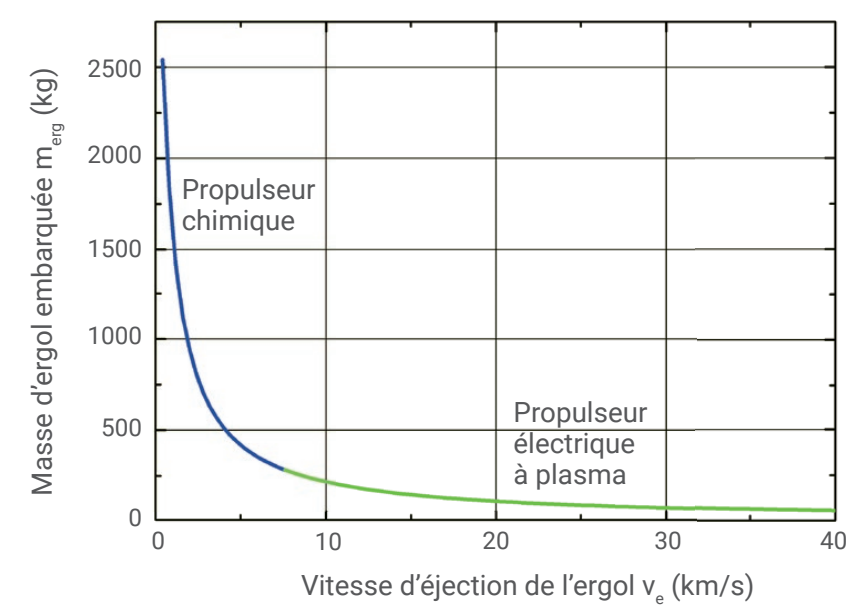

1. Masse d'ergol embarquée, $m_{\text {erg, }}$ en fonction de la vitesse d'éjection de l'ergol $v_{\mathrm{e}}$, pour un propulseur chimique et pour un propulseur à plasma.

\section{Déplacement d'un engin spatial}

Une fois séparé de son lanceur, un engin spatial (sonde d'exploration ou satellite artificiel) a besoin d'assurer un certain nombre de manœuvres. Dans le cas d'un satellite artificiel, il s'agit de l'amener sur son orbite finale, puis de l'y maintenir, corriger son attitude (pointage vers la Terre) afin qu'il effectue sa mission, et enfin l'extraire de son orbite de manière à laisser celle-ci libre à la fin de la mission. Dans le cas d'une sonde d'exploration, l'engin doit s'échapper de l'attraction terrestre, naviguer dans l'espace, puis se mettre en orbite autour de l'objet céleste étudié. À chaque mission est attribué un incrément de vitesse $\Delta v$ caractéristique de l'orbite souhaitée. À titre d'exemple, sont nécessaires des $\Delta v$ autour de $750 \mathrm{~m} / \mathrm{s}$ pour maintenir un satellite placé en orbite géostationnaire terrestre durant quinze ans, de quelques $\mathrm{km} / \mathrm{s}$ pour un transfert entre 




2. Schéma d'un propulseur de Hall à simple étage [2]. Le plasma composé d'ions et d'électrons est généré à partir d'atomes neutres de xénon injectés à l'arrière du canal en céramique et de forme cylindrique (en jaune).

l'orbite basse et l'orbite géostationnaire, et de centaines de $\mathrm{km} / \mathrm{s}$ pour une sonde voulant atteindre des planètes éloignées de 10000 unités astronomiques (distances Terre-Soleil).

Un véhicule spatial peut se mouvoir grâce à l'expulsion d'une certaine quantité d'ergol ${ }^{(a)}$ embarqué par un moteur. En écrivant la seconde loi de Newton (ou principe d'action-réaction) entre un instant initial $t$ (pour un engin spatial de masse $m_{0}$ ) et l'instant $t+\Delta t$ où l'engin spatial s'est déplacé d'une distance correspondant à un incrément de vitesse $\Delta v$, grâce à l'expulsion d'une masse d'ergol $m_{\text {erg }}$ à la vitesse d'éjection supposée constante $v_{\mathrm{e}^{\prime}}$ et en négligeant les forces extérieures s'appliquant sur celui-ci, nous aboutissons à l'équation fondamentale de la propulsion, aussi appelée équation de Tsiolkovski :

$$
m_{\mathrm{erg}}=m_{0}\left[1-\exp \left(-\Delta v / v_{\mathrm{e}}\right)\right](1) \text {. }
$$

La figure 1 illustre la variation, en fonction de la vitesse d'éjection, de la masse d'ergol $m_{\text {erg }}$ nécessaire au maintien en orbite d'un satellite de trois tonnes d'une durée de vie de quinze ans pour deux types de propulseur ("thruster" en anglais).

Le premier type de propulseur est un moteur chimique qui éjecte par exemple de l'hydrazine ${ }^{(b)}$ à des vitesses de quelques km/s, la quantité d'ergol au décollage variant alors entre $500 \mathrm{~kg}$ et 2 tonnes.

Le second type de propulseur est un moteur électrique à plasma qui éjecte des ions xénon à une vitesse entre 10 et $40 \mathrm{~km} / \mathrm{s}$, permettant de réduire la masse d'ergol à moins de $250 \mathrm{~kg}$. Cette réduction se traduit par une baisse drastique du cout du lancement (20 k€ par kg lancé dans l'espace) ou permet de lancer une charge utile plus importante. Dans cet article, nous nous intéresserons aux propulseurs à plasma.

\section{Comment générer puis accélérer des particules chargées dans un propulseur à plasma?}

Les particules chargées sont générées dans une décharge électrique par l'ionisation d'un gaz neutre, c'est-àdire la création d'un plasma d'ions positifs et d'électrons par collisions entre des électrons de haute énergie et des atomes du gaz. Ces particules chargées vont ensuite être accélérées par des champs électromagnétiques pour propulser l'engin spatial.

Une première manière d'accélérer des ions positifs est d'utiliser un système de grilles percées de trous portées à un potentiel accélérateur, dans le but de convertir cette énergie potentielle en énergie cinétique pour accélérer les ions à de grandes vitesses : c'est le moteur à grilles. Le principe de base a été démontré par E. Stuhlinger (1913-2008), puis repris par H.R. Kaufman (1926-2018). 
$>>$

Un autre concept, appelé propulseur de Hall et proposé par A. Morozov (1928-2009), est basé sur l'accélération des ions du plasma par un champ électrique axial, résultant d'une différence de potentiel continue entre une anode placée au fond d'un canal de décharge de symétrie cylindrique et une cathode placée à l'extérieur qui fournit les électrons primaires (fig. 2). Un champ magnétique, principalement radial, généré au moyen de bobines alimentées par un courant et d'un circuit ferromagnétique, a pour rôle de confiner les électrons dans le canal en une région localisée proche du plan de sortie. L'existence du champ électrique $\mathbf{E}_{\mathbf{z}}$ axial et du champ magnétique $\mathbf{B}_{\mathbf{r}}$ radial implique une dérive des électrons dans la direction azimutale $\mathbf{E}_{\mathbf{z}} \times \mathbf{B}_{\mathbf{r}}$. Ce mouvement libre des électrons ( " courant de Hall », à l'origine du nom de ce type de propulseur) augmente leur temps de résidence dans le canal et leur probabilité de collision avec les neutres. La cathode génère également des électrons nécessaires à la neutralisation du faisceau d'ions, afin d'éviter de charger électriquement l'engin spatial. Le gaz d'atomes neutres de xénon est injecté à l'arrière du canal [1]. La puissance électrique nécessaire à l'alimentation des propulseurs à plasma est fournie par des panneaux solaires.

\section{Besoins pour les missions du futur}

Parmi les technologies matures, les propulseurs de Hall et ceux à grilles occupent aujourd'hui une place prépondérante puisqu'ils ont été ou sont embarqués sur plus de $80 \%$ des 200 satellites de télécommunications placés en orbite géostationnaire depuis 1993, et qu'ils assurent la propulsion de la petite dizaine de sondes d'exploration envoyées à ce jour aux confins du système solaire [3].

Si, au-delà de la réduction du cout de la mission, la propulsion plasma offre aussi un intérêt en termes de fiabilité durant de longues missions et de variabilité en termes de performances, elle demeure encore limitée au niveau de la poussée. La poussée (exprimée en newtons) est la force exercée par le moteur : $P=d_{\mathrm{erg}} v_{\mathrm{e}}$ (où $d_{\mathrm{erg}}$ est le débit massique d'ergol exprimé en $\mathrm{kg} / \mathrm{s}$, et $v_{\mathrm{e}}$ la vitesse d'éjection exprimée en $\mathrm{m} / \mathrm{s}$ ).
Une des contraintes à bord des satellites est la taille limitée des panneaux solaires et la modeste puissance disponible qui en découle. De faibles débits d'ergol sont utilisés et le niveau de poussée ainsi obtenu est loin d'atteindre (par un facteur 10 à 30) celui des propulseurs chimiques qui, eux, ne nécessitent pas de source d'énergie extérieure. Une poussée importante est indispensable quand il est nécessaire de restreindre l'exposition du véhicule spatial dans des environnements sensibles tels que les ceintures de radiation de Van Allen qui peuvent détériorer les instruments de bord des satellites, ou lors des futurs vols habités pour réduire les risques d'irradiation de l'équipage.

Les systèmes propulsifs chimiques et électriques étaient jusqu'à récemment utilisés de manière complémentaire. Mais en 2012 puis en 2017, les succès de la " mise à poste "(c) des satellites 702SP (moteur à grille) lancé par Boeing, et Eutelsat 172B (moteur de Hall) lancé par Airbus Defense and Space, ont démontré la possibilité d'utiliser la propulsion plasma pour la mise à poste du satellite sur son orbite opérationnelle, ainsi que pour son maintien sur cette orbite [4]. Maintenant, l'accroissement de la vitesse d'éjection de l'ergol pour diminuer encore plus la consommation de carburant est devenu un enjeu crucial.

\section{Limites actuelles des moteurs à plasma conventionnels}

Au-delà des questionnements autour de la limitation actuelle de la puissance électrique disponible (l'emploi de générateurs nucléaires est envisagé dans le futur, notamment pour des missions vers Mars), les moteurs à plasma existants ne permettent pas de remplir nombre des missions envisagées, faute d'avoir un niveau de poussée suffisamment élevé quand la mission nécessite un transfert d'orbite, et une forte vitesse d'éjection de l'ergol pour le maintien à poste des satellites ou le voyage d'une sonde interplanétaire.

Pour un moteur à grilles, la poussée est proportionnelle à $[V / d]^{2} S$, où $V$ est la tension accélératrice, $d$ la distance entre les grilles et $S$ la surface d'une grille. L'augmentation de la tension accélératrice $V$ demande un système d'alimentation électrique de plus en plus complexe et lourd. De plus, une réduction de $d$ en dessous du millimètre est difficilement envisageable pour des raisons de tenue mécanique des matériaux. D'autre part, le rapport $V^{3 / 2} / d^{2}$ contrôle l'optique du faisceau d'ions et l'érosion des grilles, limitant la durée de vie du propulseur. Les surfaces $S$ actuellement réalisables ne permettent pas d'obtenir les niveaux de poussée souhaités.

Dans un propulseur de Hall, la localisation de la barrière magnétique contrôle à la fois la localisation des régions d'ionisation et d'accélération en baissant localement la conductivité des électrons, ce qui entraine une augmentation du champ électrique à cet endroit. Les essais en chambre à vide ont montré qu'un contrôle fin et indépendant de la poussée et de la vitesse d'éjection des ions ne peut être réalisé aujourd'hui. Les technologies actuelles ne répondent donc pas aux besoins du futur, par manque de versatilité.

\section{Vers plus de versatilité avec le propulseur de Hall à double étage}

Dans le propulseur dit à simple étage présenté sur la figure 2, c'est le même champ électrique qui contrôle l'extraction et l'accélération des ions d'une part, ainsi que l'ionisation du gaz d'autre part. Ceci implique que la vitesse des ions et la poussée (ionisation du gaz) ne sont pas indépendantes. On peut alors imaginer un autre type de propulseur de Hall où l'ionisation ne dépend plus uniquement des électrons émis par la cathode et accélérés par le champ électrique, mais aussi d'une source indépendante placée en amont de la barrière magnétique et de la région d'accélération. La puissance déposée dans le gaz par cette source d'ionisation pourrait alors être ajustée indépendamment de la différence de potentiel appliquée entre la cathode et l'anode. Cette configuration est appelée propulseur de Hall à double étage (DSHT pour Double Stage Hall Thruster), étant donné que l'ionisation du gaz et l'accélération des ions peuvent alors être découplées et avoir lieu dans deux étages distincts (fig. 3). 


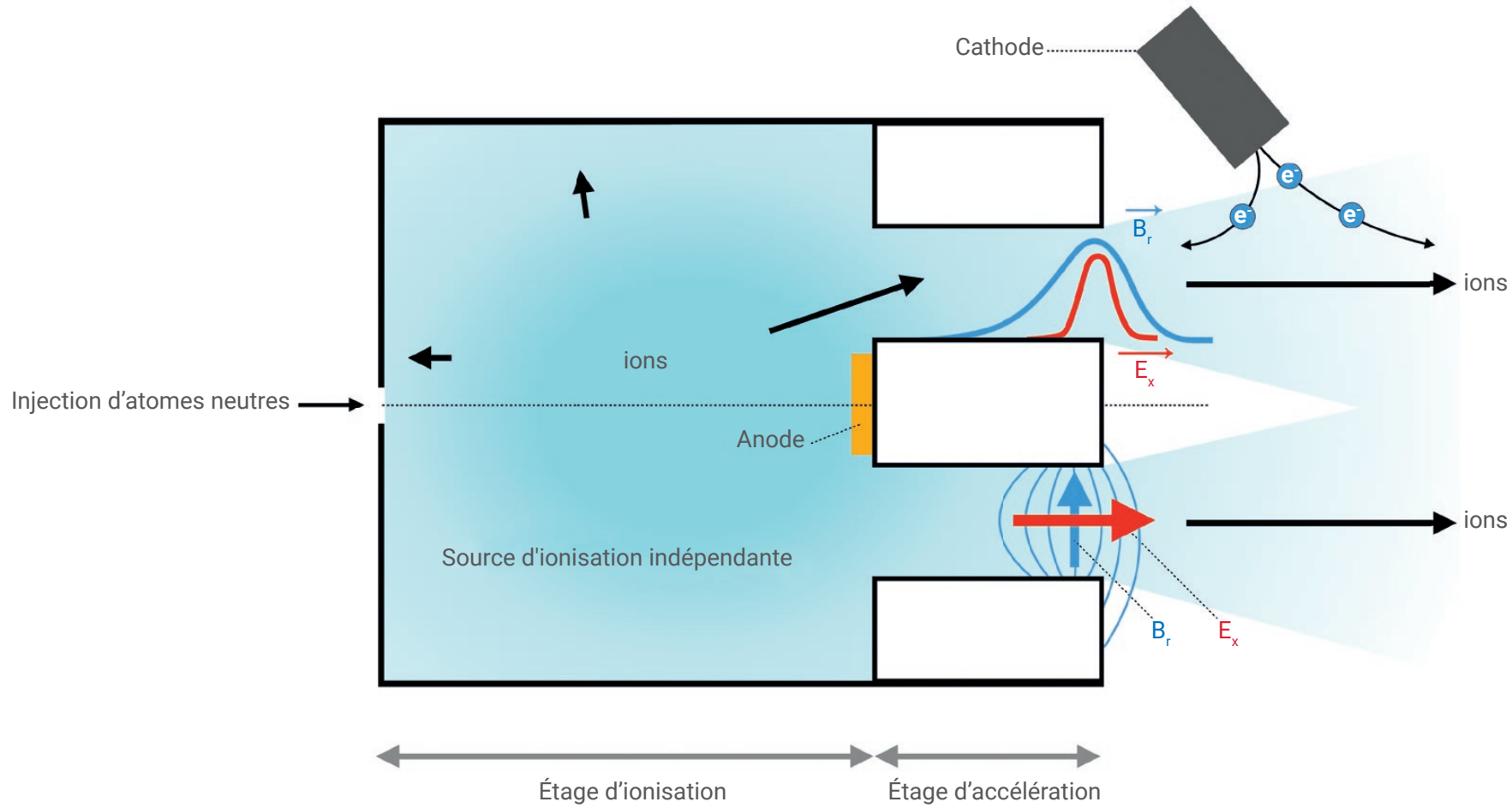

3. Représentation schématique du principe d'un propulseur de Hall à double étage. L'étage d'accélération des ions est constitué de la barrière magnétique (identique à celle du propulseur de Hall à simple étage présenté sur la figure 2). L'étage d'ionisation, constitué d'une source indépendante, est situé en amont. Les ions générés par la source doivent être guidés efficacement vers l'étage d'accélération, en limitant les pertes aux parois.

Dans un propulseur de Hall à double étage idéal, l'ionisation serait contrôlée par le débit de gaz et la puissance déposée dans le premier étage (étage d'ionisation), tandis que l'accélération serait contrôlée par la différence de potentiel appliquée entre la cathode et l'anode. Cependant, une part importante des électrons émis par la cathode sert à neutraliser le faisceau d'ions éjectés du propulseur. Ces électrons sont également accélérés par le champ électrique et peuvent contribuer en partie à l'ionisation des neutres qui n'ont pas été ionisés en amont dans le premier étage. Cela montre qu'un découplage parfait entre ionisation et accélération n'est pas possible. Afin de minimiser le rôle des électrons émis par la cathode dans l'ionisation du gaz, le champ magnétique peut être ajusté pour que le courant d'électrons soit le plus faible possible, ou la tension entre cathode et anode peut être diminuée de manière à ce que le champ électrique ne soit pas suffisant pour ioniser le gaz. Un propulseur de Hall à double étage est alors très pertinent pour des opérations où l'on cherche une faible vitesse ionique ou une forte poussée. On peut également utiliser des gaz plus légers que le xénon (par exemple l'argon), puisque pour atteindre la même impulsion spécifique (vitesse des ions éjectés) la tension appliquée entre la cathode et l'anode sera plus faible et dans certains cas insuffisante pour ioniser le gaz dans la région d'accélération.

Depuis une quinzaine d'années, plusieurs conceptions de propulseurs de Hall à double étage ont été proposées et testées. Leurs performances n'ont toutefois pas atteint les objectifs fixés, notamment ceux correspondant au cas idéal où l'ionisation du gaz et l'accélération des ions sont totalement découplées. Dans la plupart des cas, le propulseur à double étage correspond à l'assemblage d'une source d'ionisation donnée à un propulseur de Hall simple étage. Les différents concepts présentés dans la littérature ne démontrent pas clairement qu'un fonctionnement en double étage idéal est possible. Cependant, les études réalisées mettent en évidence certains points qui permettent de proposer un nouveau concept de propulseur de Hall à double étage (fig. 3) :
- la source d'ionisation (premier étage) doit être efficace et capable de générer des densités de plasma supérieures à $10^{18} \mathrm{~m}^{-3}$;

- les pertes des ions sur les parois entre le premier étage et l'étage d'accélération doivent être minimisées, au risque de perdre le bénéfice de l'ionisation dans le premier étage ;

- les ions doivent être extraits et guidés efficacement depuis la région d'ionisation vers la zone d'accélération ;

- l'ionisation doit être située dans une région immédiatement en amont de la zone d'accélération.

\section{Un nouveau concept de propulseur à double étage : ID-Hall}

Les considérations précédentes nous ont amenés à proposer un nouveau concept de propulseur de Hall à double étage. Les principales différences avec les propulseurs proposés dans la littérature concernent la nature de la source d'ionisation, ainsi que la géométrie et la configuration magnétique. Il est essentiel que ces paramètres soient optimisés de façon à 
$>>>$

ioniser efficacement le gaz et à extraire vers la région d'accélération tous les ions générés par la source d'ionisation.

Le propulseur à double étage proposé, ID-Hall pour Inductive Double stage Hall thruster, a été conçu au Laboratoire plasma et conversion d'énergie, à l'Université de Toulouse, dans ce but.

L'énergie est couplée inductivement aux électrons à l'aide d'une source d'ionisation constituée d'une bobine parcourue par un courant radiofréquence et d'aimants, et placée dans le cylindre central du propulseur (fig. 4). Cette configuration originale s'appuie sur la géométrie naturelle d'un propulseur de Hall et permet une très bonne proximité entre l'étage d'ionisation et l'étage d'accélération, contrairement à la plupart des propulseurs à deux étages.

L'étage d'ionisation possède sa propre configuration magnétique, qui 1) joue un rôle pour renforcer l'efficacité du couplage d'énergie inductif 2) confine le plasma et limite les pertes d'ions aux parois, 3) se raccorde de façon optimale à la barrière magnétique de la région d'accélération.

Plusieurs questions se posent quant à la faisabilité même du concept de double étage.

Est-il possible d'extraire et accélérer des ions, créés par une source indépendante, au travers d'une barrière magnétique?

L'injection d'un courant d'électrons à travers la barrière magnétique est nécessaire, ne serait-ce que pour neutraliser le faisceau d'ions extrait. Ces électrons peuvent donc être accélérés par le champ électrique et participer à l'ionisation du gaz. La question de la contribution relative de l'ionisation dans la source et dans la barrière, ainsi que celle de son contrôle, n'ont pas été abordées dans la littérature.

La question du transport des électrons se pose, comme dans tous les propulseurs de Hall. Dans le cas du double étage, en supposant que toute l'ionisation est faite dans le premier étage, les électrons entrants doivent alors traverser la barrière sans collisions.

La figure 5 présente des résultats de simulations obtenues à partir d'une modélisation bidimensionnelle dans le plan radial-axial [5]. Cette figure

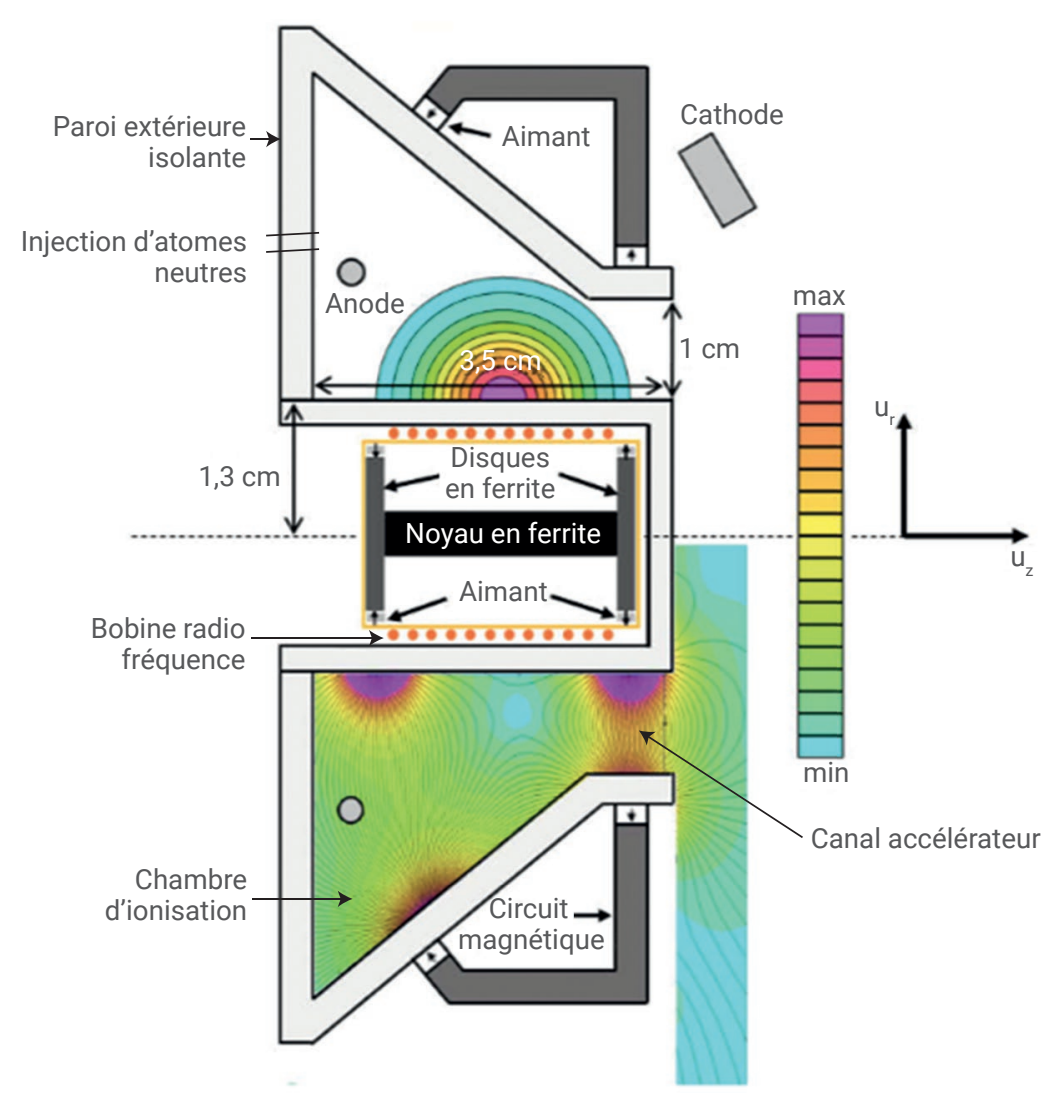

4. Vue en coupe r-z du propulseur ID-Hall. Le trait horizontal en pointillés au milieu de la figure représente l'axe central du propulseur à géométrie cylindrique. L'anode est un anneau métallique. La source d'ionisation est constituée d'une bobine radiofréquence et d'un circuit magnétique insérés dans le tube cylindrique central en quartz. Le fer doux et le ferrite associés aux aimants constituent le circuit magnétique intérieur permettant de générer la configuration magnétique représentée en haut par les lignes de champ (en traits pleins) et la puissance radiofréquence déposée (en couleur). En bas, l'intensité du champ est indiquée par des dégradés de couleurs (le violet-rouge étant le plus intense et le bleu turquoise le plus faible).

montre les distributions spatiales de la densité du plasma $n$ et de la densité d'atomes $n_{\mathrm{a}}$. Entre autres, on remarque que la densité du plasma est maximale au voisinage de la paroi extérieure de la chambre d'ionisation et décroit vers l'axe de la chambre et l'entrée du canal d'extraction. Les électrons sont confinés dans la région où le champ magnétique est parallèle aux parois (fig. 4). De plus, à partir de la simulation, on estime que les pertes d'ions aux parois sont de 2,7 A alors que le courant d'ions extrait est de 1,2 A. Ce résultat montre que les pertes d'ions ne sont pas négligeables, du fait d'une configuration magnétique non optimisée. On remarque également sur la figure 5 une déplétion des neutres (due à l'ionisation du gaz) dans un volume relativement grand de la chambre d'ionisation en amont de l'étage d'accélération, ce qui indique qu'une large part de l'ionisation s'effectue dans l'étage d'ionisation via le chauffage des électrons par la source inductive radio-fréquence, comme souhaité.

\section{Mise en évidence d'instabilités dans le propulseur ID-Hall}

L'évolution en fonction du temps du courant de décharge mesuré sur l'anode du propulseur présente des pics épisodiques espacés de $200 \mu$ s, visibles sur la courbe rose de la figure 6a (p. 30). Un pic de courant correspond à une ionisation importante du gaz, qui s'accompagne d'une déplétion des neutres dans le canal du propulseur. La zone à faible courant de décharge entre deux pics de courant est le temps nécessaire pour que le canal se remplisse à nouveau de neutres pour 


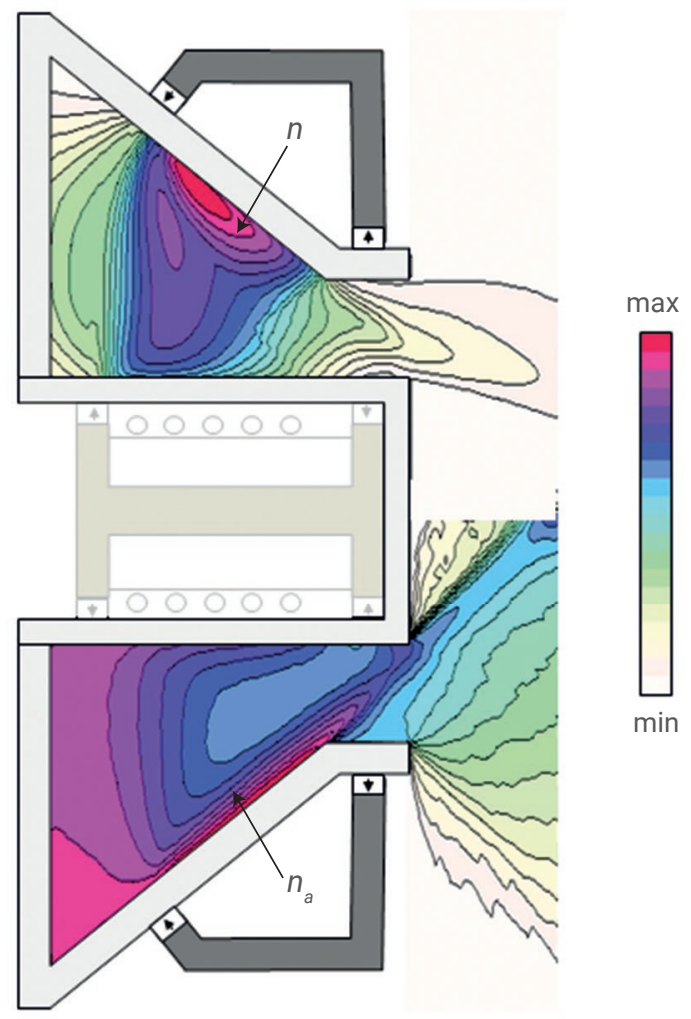

5. Propulseur ID-Hall : distributions spatiales de la densité du plasma $\boldsymbol{n}$ [maximum $6 \times 10^{18} \mathrm{~m}^{-3}$ ], et de la densité d'atomes $\mathrm{n}_{\mathrm{a}}$ [maximum : $10^{20} \mathrm{~m}^{-3}$, échelle logarithmique - 3 décades]. Tension entre cathode et anode $V_{D C}=100 \mathrm{~V}$, puissance dans la source RF de l'étage d'ionisation $P_{R F}=200 \mathrm{~W}$, débit massique de xénon $d_{\text {erg }}$ de $2 \mathrm{mg} / \mathrm{s}$.

initier une nouvelle décharge (prochain pic de courant). La fréquence de l'instabilité d'ionisation des atomes, de l'ordre de quelques $\mathrm{kHz}$, peut être reliée à la vitesse des neutres et à la longueur effective d'ionisation définie comme la distance entre la position $\mathrm{du}$ front d'ionisation dans le canal et le plan de sortie. Ce mode est appelé " mode de respiration".

Nous illustrons sur cette même figure l'évolution de la luminosité du plasma enregistrée par une caméra rapide (courbe noire). On observe une très bonne corrélation entre le pic du courant de décharge et le maximum d'émission lumineuse correspondant à une ionisation intense des atomes dans le canal.

Dans les propulseurs de Hall, d'autres types d'instabilités, liées à la dérive des électrons, peuvent apparaitre. En
RF éteinte) à l'aide d'une caméra rapide en vue de face [6]. Les images de effet, la combinaison du champ électrique $\mathbf{E}$ génère dans la direction ExB un courant de dérive fermé dû à dérive très important conduisent au sont à des échelles spatiales cent riques. Le rôle de ces instabilités sur sion du fonctionnement de ce type de propulseur [6].

Ce type d'instabilité a été observé dans le propulseur ID-Hall fonctionnant en mode simple étage (source d'ionisation direction azimutale, dont cer l'évolution de l'émission lumineuse du plasma dans la direction azimutale (fig. 6b, p. 30) ont été enregistrées à différents instants dans la phase de montée du pic de courant (les numéros des images sont reportés sur la figure 6a). Nous observons une zone lumineuse localisée, en rotation azimutale dans le sens des aiguilles d'une montre et dont l'intensité est corrélée à la montée du pic de courant de décharge. Une anode en forme d'anneau segmentée en quatre secteurs (représentés en pointillés et en différentes couleurs sur la figure 6b), a permis de suivre l'évolution temporelle des courants sur chaque secteur et de montrer que les électrons étaient majoritairement transportés lors du passage de la zone lumineuse. En effet, on observe que le courant est maximal sur le secteur en vis-à-vis du pic d'émission lumineuse et représente pour les images 2-3-4 plus de $60 \%$ du courantélectronique. À titre d'exemple, à la position 3, le maximum d'émission lumineuse se situe devant le secteur nord-ouest en vert, correspondant au maximum de courant recueilli sur ce même secteur.

D'autre part, des mesures optiques basées sur la méthode parallaxe (non présentées ici) ont permis de mettre en évidence une propagation axiale (vers le fond du propulseur) du pic d'émission lumineuse au cours d'une période du mode de respiration. Dans la phase de montée du courant de décharge (images 2-3-4), le pic lumineux est intense et localisé dans le canal, proche du plan de sortie du propulseur, tandis qu'il se déplace vers le fond du canal (tout en se propageant dans la direction azimutale), se rapprochant de l'anode, lorsque le courant est maximal (images 5-6).

Le fonctionnement en double étage (source d'ionisation active) entraine la suppression des deux types d'instabilités précédentes dès quelques dizaines de watts absorbés par le plasma. Ceci est certainement dû à la modification de la zone d'ionisation du gaz.

\section{Conclusions et perspectives}

Un nouveau concept de propulseur à courant de Hall à double étage a été proposé et étudié au laboratoire Laplace à l'Université de Toulouse. Une source plasma inductive ( $1^{\mathrm{er}}$ étage) 

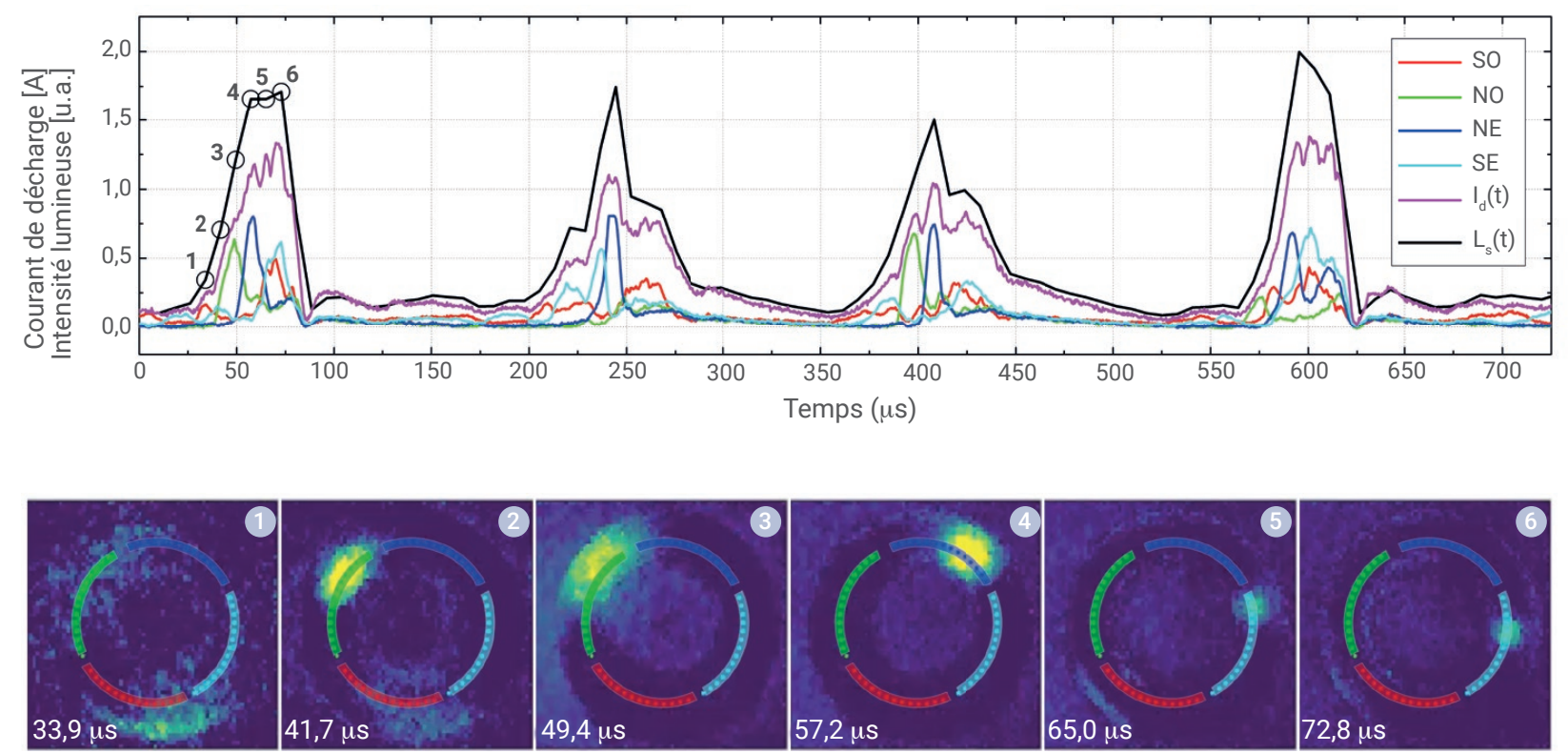

6. Instabilités observées dans le propulseur ID-Hall en mode simple étage (tension entre la cathode et l'anode $V_{D C}=120 \mathrm{~V}$, débit massique de xénon $d_{\text {erg }}=0,6 \mathrm{mg} / \mathrm{s}$ ).

(a) Évolution temporelle de la luminosité du plasma (courbe noire), des courants de décharge mesurés sur l'anode du propulseur (courbe rose) et sur quatre segments de cette anode (les couleurs des courbes correspondent à celles des secteurs de l'anode représentés sur les images de la figure 6b).

(b) Images du propulseur ID-Hall vu de face, obtenues par caméra rapide, après traitement numérique, prises à six instants consécutifs dans la phase de montée du premier pic de luminosité du plasma (courbe noire de la figure 6a). On observe une zone lumineuse qui se déplace dans le sens horaire au cours du temps.

>>>

permettant d'ioniser le gaz est placée en amont de la barrière magnétique accélératrice pour les ions ( $2^{\mathrm{e}}$ étage). Même si la validité du concept de double étage a été prouvée (il est possible d'accélérer des ions, créés par une source indépendante, au travers d'une barrière magnétique), les mesures ainsi qu'une modélisation bidimensionnelle ont mis en évidence que les pertes étaient toutefois importantes et que la configuration magnétique devait être optimisée. La zone d'ionisation doit être placée au plus près de la zone d'accélération. Dans ce but, nous avons conçu un nouveau propulseur à double étage (ID-Hall) qui est à l'étude, et dans lequel l'ionisation du gaz est localisée directement dans la barrière accélératrice.

Une gamme assez large d'instabilités peut apparaitre au sein du plasma des propulseurs de Hall, parmi lesquelles une instabilité de rotation d'une fréquence de quelques $\mathrm{kHz}$, qui se propage azimutalement dans la direction des champs croisés ExB. Nous avons montré que cette instabilité est couplée à une instabilité axiale d'ionisation. Au cours d'une période d'ionisation, l'instabilité de rotation présente un mouvement hélicoïdal depuis le plan de sortie du propulseur vers l'anode située au fond du canal.

Au-delà des propulseurs de Hall, ce type d'instabilité (et bien d'autres) se retrouve dans d'autres dispositifs plasmas en champs électrique et magnétique croisés en présence de dérive fermée électronique. L'identification et la compréhension des mécanismes à l'origine de ces structures sont aujourd'hui des défis d'importance majeure pour la communauté des physiciens des plasmas. I

(a) Un ergol est une substance employée seule ou associée à d'autres substances pour fournir de l'énergie. Cet ergol peut être sous forme solide ou liquide (par exemple l'hydrazine) dans les propulseurs chimiques, ou gazeuse (par exemple le xénon) dans un propulseur à plasma.

(b) L'hydrazine (de formule $\mathrm{N}_{2} \mathrm{H}_{4}$ ) est un des combustibles liquides les plus utilisés.

(c) Mettre à poste : rejoindre la trajectoire recherchée pour sa mission.
1. S. Mazouffre, «Les propulseurs à plasma : une technologie spatiale d'avant-garde », Reflets de la Physique 14 (2009) 15-19.

2. A. Guglielmi, « Nouveau concept de propulseur à courant de Hall », Thèse de doctorat de l'Université Paul Sabatier, Toulouse (2020).

3. D. Lev et al., "The Technological and Commercial Expansion of Electric Propulsion in the Past 24 Years", presented at the $35^{\text {th }}$ International Electric Propulsion Conference, Atlanta, GA, 2017.

4- I. Levchenko et al., "Perspectives, frontiers, and new horizons for plasma-based space electric propulsion", Physics of Plasmas 27 (2020) 020601

5. L. Dubois et al., "ID-HALL, a new double stage Hall thruster design. I. Principle and hybrid model of ID-HALL", Physics of Plasmas 25 (2018) 093503

6• J.P. Boeuf, "Tutorial: Physics and modeling of Hall thrusters", Journal of Applied Physics 121 (2017) 011101. 